Recebido em 12/2013. Aceito para publicação em 09/2014.

\title{
AGENTES FísICOS EM UNIDADES BÁSICAS DE SAÚDE: POTENCIALIDADE DE RISCOS OCUPACIONAIS
}

\section{PHYSICAL AGENTS IN BASIC HEALTH UNITS: POTENTIAL OCCUPATIONAL RISKS}

\author{
Natália Pereira Marinelli ${ }^{1}$ \\ Maria Belén Salazar Posso² \\ Tarciso Marinelli Filho ${ }^{3}$
}

Resumo: Os ambientes de trabalho em que atuam os profissionais de saúde, como as Unidades Básicas de Saúde da Família (UBSF), concentram, por sua natureza, uma série de riscos que podem trazer diversos agravos à saúde e acometem, em especial, trabalhadores de Enfermagem. Este estudo teve o objetivo de identificar e descrever as potencialidades dos agentes físicos, como riscos ocupacionais nas Unidades Básicas de Saúde (UBS). Trata-se de uma revisão de literatura integrativa, por meio de pesquisa em artigos científicos publicados em periódicos nacionais e internacionais nos últimos 10 anos, encontrados nas bases de dados LILACS, IBECS, Biblioteca Cochrane, SciELO e MEDLINE pelo PUBMED. Como resultado, constatou-se que, dos 156 riscos ocupacionais físicos citados, apenas sete eram específicos para UBS: a temperatura, o ruído, vibrações, pressões anormais, radiações, iluminação, ventilação e umidade. Outros fatores que podem ser incluídos são o espaço físico inadequado, a disposição da mobiliário, além da ausência de adaptação dos espaços internos e externos da UBS para pessoas com necessidades especiais.

Palavras-chave: riscos ocupacionais; centros de Saúde; Saúde da Família; enfermagem.

Abstract: The working environments in which health professionals work, such as basic family health units (BFHU), concentrate by their nature a number of risks that can be harmful to health and affect particularly nurses. This study aimed to identify and describe the potential of physical agents as occupational hazards in Basic Health Units (BHUs). This integrative literature review was conducted through research of published scientific articles in national and international journals in the last 10 years, found in the databases LILACS, IBECS, Cochrane Library, SciELO, MEDLINE, in PubMed. As a result, we found that of the 156 physical occupational hazards cited, only seven were specific to BHUs: temperature, noise, vibration, abnormal pressures, radiation, lighting, ventilation, and humidity. Other factors that may be included are the inadequate physical space, furniture layout, in addition to the lack of adaptation of internal and external spaces of BHUs for people with special needs.

Keywords: occupational risks; Health centers; Family Health; nursing.

\footnotetext{
1 Mestra em Bioengenharia - Universidade do Vale do Paraíba - Univap, Brasil e Docente do Colégio Técnico da Universidade Federal do Piauí - UFPI, Brasil. E-mail: enfnatmarinelli@hotmail.com.

${ }^{2}$ Doutora em Enfermagem - Universidade de São Paulo - USP, Brasil e Docente da Univap. E-mail: mbelen@terra.com.br.

${ }^{3}$ Especialista em Saúde da Família e Saúde Pública e Enfermeiro do Hospital Municipal de Zé Doca - MA, Brasil. E-mail: tfmarinelli@hotmail.com.
} 


\section{INTRODUÇÃO}

Nas décadas de 80 e 90 do século XX, pesquisadores já estudavam as repercussões do processo de trabalho na saúde dos profissionais da área hospitalar, com o objetivo de identificar os riscos ocupacionais a que esses trabalhadores se encontravam expostos. Atualmente, essas discussões têm como foco a organização do trabalho, sendo este capaz de influenciar, de modo específico, os trabalhadores de saúde (SÊCCO; ROBAZZI, 2003; SOUSA; CAMPOS, 2008).

Todas as atividades do ser humano, inclusive aquelas destinadas ao lazer, envolvem riscos, cuja incidência pode vir a ser de maior ou menor gravidade, dependendo das suas características específicas (POSSO, 1988). Dessa forma, o ambiente de trabalho tem seus riscos, sendo importante avaliar, continuamente, suas fontes geradoras, para que o gerenciamento e medidas de prevenção sejam implementados de maneira bem sucedida (BENEDETT; FERRAZ; POSSO, 2009). Ainda, como afirma Nunes (2009), os riscos devem ser vistos e analisados sob o prisma da potencialidade, permitindo que se identifiquem as fontes potenciais de riscos e de agravos à saúde, favorecendo a adoção de medidas preventivas de segurança.

Diante disso, parece lícito afirmar que o risco está presente, cotidianamente, na atividade dos profissionais de saúde. Existem várias significações de risco devido às formas como se apresentam, variando de acordo com seu seu nível de abrangência, maior ou menor gravidade, e os possíveis agravos que possam advir à saúde do ser humano.

Assim, risco é a possibilidade de ocorrência de perigo, é o medo ou receio de qualquer coisa, eventos ou fatos que possam causar algum dano ou mal (POSSO, 1988), complementando-se com o conceito transmitido pela Organização Internacional do Trabalho (OIT, 1989) que define risco como:

a possibilidade real ou potencial compreendendo situações, episódios, eventos, fatos imprevistos, incertos, capazes de causar dano, lesão e/ou morte, ou perdas patrimoniais, suspensão temporária do processo de trabalho ou, ainda, de afetar a comunidade e/ou o meio ambiente.

Ao analisar o contexto atual, verifica-se que é muito frequente entre os trabalhadores de saúde, principalmente da área de Enfermagem, ganhar a vida correndo de um posto de trabalho para o outro, sem questionar os riscos a que estão expostos em seu ambiente laboral. Riscos ocupacionais originam-se de atividades laborais insalubres que podem trazer sérios danos à saúde dos trabalhadores (CASTRO; FARIAS, 2008). Assim, Mauro et al. (2004) e Nunes (2009) afirmam que risco é definido como uma ameaça possível de causar uma reação adversa à saúde das pessoas a ele expostas ou como a possibilidade de agravos na saúde física e psíquica, em diversos campos: moral, intelectual cultural e espiritual do ser humano, Sendo que qualquer que seja a origem, a potencialidade do risco deve ser prevenida, adotando-se medidas de proteção.

Os funcionários que trabalham em Unidades Básicas de Saúde da Família (UBSF) estão expostos a riscos ambientais que podem ser classificados, de acordo com o seu agente 
etiológico, em físicos, químicos, biológicos e psicológicos (BRASIL, 1977; 2004; 2005; CAMPANHOLE, 2004; SILVA; FELLI, 2002; POSSO; SANT'ANNA, 2007). Acrescentem-se a esses os riscos mecânicos e ambientais (BRASIL, 1994; COSTA, 2003), ressaltando-se, ainda, os riscos legais, tão presentes no cotidiano desses profissionais (POSSO; COSTA, 1998).

Os riscos ocupacionais que acometem os trabalhadores de saúde são oriundos de fatores físicos gerados pelas diversas formas de energia a que possam estar expostos. São exemplos dessas situações: o ruído, a iluminação, a temperatura, entre outros. Os riscos físicos tem a vantagem de serem mensurados por equipamentos, podendo, consequentemente, ser evitados ou corrigidos com certa facilidade (MARINHO, 2004).

Os ambientes de trabalho nos quais atuam os profissionais de saúde, como as UBSF, concentram, por sua natureza, uma série de riscos que podem trazer diversos agravos à saúde para os profissionais que lá trabalham, especialmente, para os trabalhadores de Enfermagem (SILVA; FELLI, 2002). A Estratégia Saúde da Família (ESF) desenvolvida pelo Ministério da Saúde, em 1994, é um modelo de atenção à saúde eficaz, eficiente e adequado ao fortalecimento da Atenção Básica $(A B)$ no país, sendo a porta de entrada do SUS e o eixo central da organização do sistema. Engloba um conjunto de ações de promoção da saúde, prevenção de agravos e tratamento, reabilitação e manutenção da saúde da população adscrita, buscando concretizar os princípios do SUS: Integralidade, Universalidade e Participação Social (BRASIL, 2008). As UBS caracterizam-se por serem estudadas por "picos de movimento", sendo consideradas ambientes estressantes pelos trabalhadores de saúde (CHIODI; MARZIALE, 2006).

O presente trabalho teve o objetivo de apresentar as potencialidades dos agentes físicos como geradores de riscos ocupacionais nas Unidades Básicas de Saúde como relatados em revisão de literatura.

\section{METODOLOGIA}

Trata-se de um estudo elaborado a partir do método descritivo-exploratório, associado à revisão integrativa da literatura (MENDES; SILVEIRA; GALVÃO, 2008); com abordagem quantitativa mediante às informações extraídas de artigos científicos publicados em periódicos nacionais e internacionais e banco de teses nos últimos 10 anos, encontrados nas bases eletrônicas de dados Literatura Latinoamericana e do Caribe em Ciências da Saúde, Índice Bibliográfico Espanhol em Ciências da Saúde, Medical Literature Analysis na Retrieval Sistem Online, Biblioteca Cochrane, Scientific Eletronic Library pelo PUBMED sobre riscos físicos em UBSF. Ainda, adotaram-se as particularidades de cada base de dados combinadas com as expressões booleanas $A N D / Y / E$, sendo que estas permitem, obrigatoriamente, obter resultados com as duas palavras selecionadas e OR/O/U/OU que permitem encontrar endereços com uma das palavras e/ou a adição de duas ou mais palavras; neste caso, digitadas nos idiomas inglês, espanhol e português. As expressões podendo ser ligadas 
mediante operadores (como AND/Y/E, OR/O, U/OU), para possibilitar a obtenção de resultados mais completos.

Os dados referentes aos estudos captados foram registrados numa ficha estruturada de catalogação de dados bibliográficos, composta dos seguintes itens: título do artigo, dissertação e tese, autoria, ano de publicação, periódico, base de dados, tipo de estudo, tipo de risco estudado e conclusão.

O levantamento bibliográfico, para a realização deste estudo, foi realizado em bibliotecas virtuais, seguindo uma ordem cronológica. Foram utilizadas as seguintes palavraschave, selecionadas de acordo com os descritores de saúde, nos idiomas: português, espanhol e inglês, como se vê a seguir:

Riscos Ocupacionais, Centros de Saúde; Saúde da Família, Enfermagem (para Língua Portuguesa);

Riesgos ocupacionales; Centros de Salud; Salud de la Familia, Enfermería (para Língua Espanhola);

Occupational Risks; Health Centers; Family Health; Nursing (para Língua Inglesa).

Foram incluídos artigos científicos, teses e dissertações que abordassem os riscos físicos ocupacionais presentes no local de trabalho de profissionais de Enfermagem em Unidades Básicas de Saúde nos idiomas português, espanhol e inglês.

Realizou-se uma leitura prévia para seleção das obras, destacando-se as ideias principais para a leitura seletiva. Após a seleção, fez-se a leitura detalhada com o intuito da apreensão do cerne do conteúdo, ampliando-se o conhecimento sobre o assunto e permitido que emergissem as categorias: presença de riscos físicos no ambiente de trabalho na assistência à saúde, inadequação de ambiente de trabalho em UBS e necessidade de esclarecimento sobre riscos ocupacionais a que estão expostos os trabalhadores de saúde.

\section{RESULTADOS}

Em relação ao delineamento da pesquisa, a análise dos estudos selecionados, fundamentou-se em Polit, Beck e Hungler (2004) e LoBiondo-Wood e Haber (2001). Assim, a análise dos dados extraídos foi realizada de forma descritiva, possibilitando reunir o conhecimento produzido sobre o tema explorado na revisão. A Figura 1 apresenta os sete artigos selecionados de acordo com as categorias que emergiram da análise textual, organizados de acordo com o artigo, a base de dados, autor, título, periódico, ano da publicação, tipo de estudo, conclusão do autor(es). Das 156 obras selecionadas, observou-se que 20 artigos (12,82\%) abordavam os diversos riscos ocupacionais, e apenas sete $(3,85 \%)$, também demonstrados na Figura 1, abordavam os riscos físicos, propriamente ditos, nas UBS. Para facilitar a identificação dos estudos incluídos, foram nomeados com a letra $A$ e numerados, sequencialmente, de AP1 a AP7 (Figura 1). Incluiram-se dois artigos completos de anais de congresso e jornada que eram específicos quanto ao objeto do estudo (AP2 e AP3), que podem ser vistos também na mesma figura. 


\begin{tabular}{|c|c|c|}
\hline \multirow[t]{3}{*}{$\begin{array}{l}\text { Artigo } \\
\text { A P1 }\end{array}$} & $\begin{array}{l}\text { Base de Dados } \\
\text { SCIELO }\end{array}$ & $\begin{array}{l}\text { Autor } \\
\text { BESSA, M. E. P. et al. }\end{array}$ \\
\hline & $\begin{array}{l}\text { Título } \\
\text { Riscos ocupacionais do enfermeiro atuante na } \\
\text { estratégia saúde da família. }\end{array}$ & $\begin{array}{l}\text { Periódico } \\
\text { Rev. Enf. UERJ., v.18, n.4, p.644-9. } 2010 .\end{array}$ \\
\hline & $\begin{array}{l}\text { Tipo de Estudo } \\
\text { Revisão Bibliográfica }\end{array}$ & $\begin{array}{l}\text { Conclusão do(s) autor(es) } \\
\text { Os autores concluem que os enfermeiros estão expostos a } \\
\text { riscos físicos (temperatura elevada e ambiente pouco } \\
\text { iluminado); ergonômicos (mobiliário inadequado para a } \\
\text { realização das atividades) e acidentes. } \\
\text { Ainda, que os riscos físicos são pouco explorados. }\end{array}$ \\
\hline \multirow[t]{3}{*}{$\begin{array}{c}\text { Artigo } \\
\text { A P2 }\end{array}$} & $\begin{array}{l}\text { Base de Dados } \\
\text { BVS/Anais/Instituto Federal de Ciência e } \\
\text { Tecnologia-BA }\end{array}$ & $\begin{array}{l}\text { Autor } \\
\text { RIOS, J. C. C.; VARELA, C. D. S. }\end{array}$ \\
\hline & $\begin{array}{l}\text { Título } \\
\text { Riscos ambientais em unidade de saúde da } \\
\text { família. }\end{array}$ & $\begin{array}{l}\text { Periódico } \\
\text { Anais da III Jornada Científica e Tecnológica do Oeste } \\
\text { Baiano. } 2010 .\end{array}$ \\
\hline & $\begin{array}{l}\text { Tipo de Estudo } \\
\text { Revisão Bibliográfica }\end{array}$ & $\begin{array}{l}\text { Conclusão do(s) autor(es) } \\
\text { Demonstram a necessidade da contextualização do } \\
\text { ambiente de trabalho e sua relação com a Saúde do } \\
\text { Trabalhador, sendo de fundamental importância o } \\
\text { esclarecimento sobre os riscos aos quais estão expostos os } \\
\text { trabalhadores, ressaltando a necessidade de treinamento e } \\
\text { conscientização de práticas seguras e mais importante, o } \\
\text { fornecimento dos EPI's. }\end{array}$ \\
\hline \multirow[t]{3}{*}{$\begin{array}{c}\text { Artigo } \\
\text { A P3 }\end{array}$} & $\begin{array}{l}\text { Base de Dados } \\
\text { BVS/ANAIS/ABEn }\end{array}$ & $\begin{array}{l}\text { Autor } \\
\text { SOARES, A. D.; COSTA, C. C. C.; RICARTE, F. M. C. }\end{array}$ \\
\hline & $\begin{array}{l}\text { Título } \\
\text { Trabalho em saúde: analisando os riscos } \\
\text { ambientais em um centro de saúde da família. }\end{array}$ & $\begin{array}{l}\text { Periódico } \\
\text { Anais do 61ํㅡㄹ CBEn Trabalho } 571 \text { - 1/2p.6323. } 2009 .\end{array}$ \\
\hline & $\begin{array}{l}\text { Tipo de Estudo } \\
\text { Pesquisa de campo }\end{array}$ & $\begin{array}{l}\text { Conclusão do(s) autor(es) } \\
\text { Os riscos físicos, estiveram presentes em } 60 \% \text { dos setores. } \\
\text { Salientando-se o ruído, o calor e a umidade. Os riscos } \\
\text { ergonômicos mostraram-se como um grande problema, } \\
\text { visto que, em } 70 \% \text { dos setores os arranjos físicos eram } \\
\text { deficientes. Já os riscos de acidentes ou mecânicos } \\
\text { estavam presentes em } 50 \% \text { dos setores como possibilidade } \\
\text { de choques elétricos, arranjos físicos deficientes, máquinas } \\
\text { e material perfurocortante sem proteção, dentre outros. }\end{array}$ \\
\hline
\end{tabular}




\begin{tabular}{|c|c|c|}
\hline \multirow[t]{3}{*}{$\begin{array}{c}\text { Artigo } \\
\text { A P4 }\end{array}$} & $\begin{array}{l}\text { Base de Dados } \\
\text { SCIELO }\end{array}$ & $\begin{array}{l}\text { Autor } \\
\text { SIQUEIRA, F. C. V. et al. }\end{array}$ \\
\hline & $\begin{array}{l}\text { Título } \\
\text { Barreiras arquitetônicas a idosos e portadores } \\
\text { de deficiência física: um estudo epidemiológico } \\
\text { da estrutura física das unidades básicas de } \\
\text { saúde em sete estados do Brasil }\end{array}$ & $\begin{array}{l}\text { Periódico } \\
\text { Ciência e Saúde Coletiva, v. 14, n.1, p.39-44,2009. }\end{array}$ \\
\hline & $\begin{array}{l}\text { Tipo de Estudo } \\
\text { Pesquisa de Campo }\end{array}$ & $\begin{array}{l}\text { Conclusão do(s) autor(es) } \\
\text { O estudo mostra que cerca de } 60 \% \text { das UBS foram } \\
\text { classificadas como inadequadas para o acesso de idosos e } \\
\text { portadores de deficiências. A presença de degraus, a falta } \\
\text { de corrimãos, rampas, banheiros adaptados para os } \\
\text { cadeirantes e salas de espera inadequadas às } \\
\text { necessidades foram uma constante. }\end{array}$ \\
\hline \multirow[t]{3}{*}{$\begin{array}{c}\text { Artigo } \\
\text { A P5 }\end{array}$} & $\begin{array}{l}\text { Base de Dados } \\
\text { SCIELO }\end{array}$ & $\begin{array}{l}\text { Autor } \\
\text { CHIODI, M. B.; MARZIALE, M. H. P. }\end{array}$ \\
\hline & $\begin{array}{l}\text { Título } \\
\text { Riscos ocupacionais para trabalhadores de } \\
\text { unidades básicas de saúde: revisão } \\
\text { bibliográfica. }\end{array}$ & $\begin{array}{l}\text { Periódico } \\
\text { Acta Paul. Enferm., v.19, n.2, p.212-217, } 2010 .\end{array}$ \\
\hline & $\begin{array}{l}\text { Tipo de Estudo } \\
\text { Revisão Bibliográfica }\end{array}$ & $\begin{array}{l}\text { Conclusão do(s) autor(es) } \\
\text { Mostram que os riscos físicos foram abordados em } 58.3 \% \\
\text { estudos e, seguidos pelos riscos químicos }(50,0 \%) \text { e por } \\
\text { fatores relacionados a condições ergonômicas }(33,3 \%) \text {. }\end{array}$ \\
\hline \multirow[t]{3}{*}{$\begin{array}{c}\text { Artigo } \\
\text { A P6 }\end{array}$} & $\begin{array}{l}\text { Base de Dados } \\
\text { LILACS }\end{array}$ & $\begin{array}{l}\text { Autor } \\
\text { FARIAS, S. N. P.; ZEITOUNE, R. C. G. }\end{array}$ \\
\hline & $\begin{array}{l}\text { Título } \\
\text { Riscos no trabalho de enfermagem em um } \\
\text { centro municipal de saúde. }\end{array}$ & $\begin{array}{l}\text { Periódico } \\
\text { Rev. Enf. UERJ, v.13, n.2, p.167-74, } 2005 .\end{array}$ \\
\hline & $\begin{array}{l}\text { Tipo de Estudo } \\
\text { Pesquisa de Campo }\end{array}$ & $\begin{array}{l}\text { Conclusão do(s) autor(es) } \\
\text { Identificaram que as cargas biológicas, físicas, mecânicas, } \\
\text { fisiológicas e psíquicas foram as mais citadas entre os } \\
\text { profissionais e também a precária utilização de proteção por } \\
\text { parte dos mesmos. }\end{array}$ \\
\hline \multirow[t]{3}{*}{$\begin{array}{c}\text { Artigo } \\
\text { A P7 }\end{array}$} & $\begin{array}{l}\text { Base de Dados } \\
\text { SCIELO }\end{array}$ & $\begin{array}{l}\text { Autor } \\
\text { SILVA, R. C. G.; FELLI, V. E. A. }\end{array}$ \\
\hline & $\begin{array}{l}\text { Título } \\
\text { Um estudo comparativo sobre a identificação } \\
\text { dos riscos ocupacionais por trabalhadores de } \\
\text { enfermagem de duas unidades básicas de } \\
\text { saúde do município de São Paulo. }\end{array}$ & $\begin{array}{l}\text { Periódico } \\
\text { Rev. Esc. Enferm. USP, v.36, n.1, p.18-24, } 2002 .\end{array}$ \\
\hline & $\begin{array}{l}\text { Tipo de Estudo } \\
\text { Pesquisa de Campo }\end{array}$ & $\begin{array}{l}\text { Conclusão do(s) autor(es) } \\
\text { Mostram que os trabalhadores de Enfermagem citaram os } \\
\text { riscos físicos por queimaduras na manipulação de } \\
\text { autoclaves; Mecânicos - pelos acidentes com objetos } \\
\text { pérfuro-cortantes, pisos escorregadios e quedas e agressão } \\
\text { física; por parte de pacientes. }\end{array}$ \\
\hline
\end{tabular}

Figura 1 - Artigos selecionados de acordo com as categorias que emergiram da análise textual. 
Os riscos ocupacionais físicos citados foram: a temperatura, o ruído, vibrações, pressões anormais, radiações, iluminação, alterações da ventilação e umidade. Outros fatores que podem ser observados são espaço físico inadequado, a disposição inadequada do mobiliário, favorecendo agravos à saúde advindos de riscos ergonômicos, além da ausência de adaptação dos espaços internos e externos da UBS para pessoas com necessidades especiais, que dificultam o transporte dessas pessoas pelos funcionários que, também, ficam sujeitos a riscos de quedas. Vale, também, destacar a importância de esclarecimento sobre os riscos a que estão expostos os trabalhadores e treinamento e conscientização de práticas de trabalho seguras.

\section{DISCUSSÃO}

A ESF assume o compromisso de prestar assistência universal, integral, equânime, contínua e, acima de tudo, resolutiva à população, tanto na unidade de saúde quanto no domicílio, sempre de acordo com as suas necessidades, identificando os fatores de risco e intervindo de maneira adequada, configurando-se em um novo modelo para a estruturação dos serviços de saúde, para a relação com a comunidade e, também, entre os vários níveis de complexidade assistencial (BRASIL, 2001).

Este estudo possibilitou a reflexão sobre a exposição do trabalhador de Enfermagem que desempenha suas atividades em UBS aos riscos físicos. Identificou, ainda, os diversos riscos físicos incidentes em UBS, evidenciados pelos autores apresentados, tais como: ruído, temperatura elevada, baixa umidade, choques elétricos, arranjos físicos deficientes, máquinas e material perfuro-cortante sem proteção (AP1, AP3, AP4 e AP7).

Os processos de trabalho e produção, nos quais o trabalhador participa como agente, podem-se compor em fatores determinantes para o desgaste de sua saúde. A inadequação das condições de trabalho nas UBS, quando estas funcionam de forma improvisada ou indesejável, é caracterizada pela maior exposição dos trabalhadores aos agentes de riscos ocupacionais - RO (DALRI; ROBAZZI; SILVA, 2010).

Já, os riscos de acidentes são aqueles inerentes a cada ambiente de trabalho, como, por exemplo, risco de queda decorrente de pisos ou iluminação inadequados (SILVA; ZEITOUNE, 2009). No ambiente laboral, o risco ocupacional pode estar oculto, por falta de conhecimento, ou de informação, situação em que o trabalhador sequer suspeita de sua existência (REZENDE, 2003).

Marinho (2004) afirma que, se o risco diz respeito ao ambiente de trabalho, incluem-se, aí, as altas ou baixas temperaturas, a exposição à radiação ionizante e não ionizante, os níveis inadequados de iluminação e ruído e o uso de mobília e equipamentos sem preocupação ergonômica, o que causa desconforto e vários problemas musculares e esqueléticos.

Cargas físicas são aquelas a que os trabalhadores se expõem quando submetidos a 
condições inadequadas de iluminação, ventilação e umidade, a mudanças bruscas de temperatura, à vibração, ao ruído, a incêndios e choques elétricos e à radiação ionizante (SILVA; FELLI, 2002). Em seu estudo, Rios e Varela (2010) afirmam que os riscos físicos são decorrentes das altas temperaturas ambientais, principalmente em centros de material e esterilização, provenientes de autoclaves e estufas, sendo associados à ventilação inadequada durante o trabalho nas UBS.

Silva e Felli (2002), ao compararem riscos ocupacionais entre duas UBS observaram que a frequência de exposição aos riscos físicos, segundo a percepção dos trabalhadores de Enfermagem em uma das UBS, foi de $8 \%$, enquanto que os entrevistados da outra UBS não citaram esse tipo de riscos (AP7 na Figura 1).

Os riscos físicos, apesar de grande relevância, pouco são discutidos na literatura. Dentre os encontrados, estão a exposição à iluminação precária, dificultando a realização de procedimentos; a falta de arejamento nos consultórios de Enfermagem, tornando esse ambiente impróprio para o trabalho; e as instalações elétricas inadequadas, podendo ocasionar choques elétricos, especialmente durante a realização da citologia oncótica, já que há a necessidade de utilização do foco de luz elétrica (BESSA et al., 2010).

Farias e Zeitoune (2005), ao realizarem uma pesquisa com os trabalhadores de Enfermagem em um centro municipal de saúde (AP6), observaram que a planta física apresentava restrição de espaço, portanto encontrava-se inadequada em relação às recomendações ministeriais para tal ambiente (BRASIL,1994; 2002), sendo que o mobiliário disposto de forma imprópria interferia no atendimento da clientela, prejudicando, ainda mais, a relação espaço x quantitativo de mobiliário e, por conseguinte, a assistência prestada.

A ausência de rampas e a falta de corrimãos, nas UBS (AP4), foi outro problema referido pelas equipes, em um estudo feito por Siqueira et al. (2009), realizado em 240 UBS, de 41 municípios de 7 estados do país, demonstrando o despreparo e falta de adaptação para pessoas com necessidades especiais, apresentando um risco físico referente à estrutura física da UBS. Esse artigo foi incluído, nesta revisão, pela dificuldade que essa situação acarreta no momento de acompanhar, movimentar e transportar o cliente com necessidades especiais, associando-se tal situação ao tipo de piso, na maioria das UBS, que costuma ser escorregadio.

É interessante destacar, dentre os trabalhos publicados, que os riscos físicos foram evidenciados em todas as sete pesquisas, ressaltando a importância de se observar esse RO, que pode causar sérios danos à saúde do trabalhador, sendo, no entanto, esse assunto tão pouco explorado pelos estudiosos de Enfermagem.

\section{CONCLUSÃO}

Dessa forma, pode-se concluir que os riscos físicos no ambiente do trabalho em saúde devem ser mapeados, corrigidos e prevenidos, pois influenciam na segurança dos 
profissionais durante suas atividades laborais e, consequentemente, na qualidade da assistência.

É fato que a literatura pouco aborda os riscos físicos, sendo tal fato preocupante, necessitando ser melhor investigado, para que medidas de prevenção e controle sejam elaboradas para garantir um ambiente saudável para os trabalhadores de saúde.

Foram incluídos, na presente revisão, sete artigos que identificaram os riscos físicos mais expressivos presentes em UBS, destacando-se ruído, altas temperaturas, choques elétricos, arranjos físicos deficientes e inadequados, pisos escorregadios, Destaca-se a importância de esclarecimento sobre os riscos a que estão expostos os trabalhadores, treinamento e conscientização de práticas seguras.

\section{REFERÊNCIAS}

BENEDETT, A.; FERRAZ, L.; POSSO, M. B. S. Os riscos de acidentes de trabalho em unidades de terapia intensiva neonatal. In: TONDIN, C. F. (Org.). Contribuições para a humanização do trabalho. Chapecó: Sinproeste, 2009. pp. 236-248.

BESSA, M. E. P. et al. Riscos ocupacionais do enfermeiro atuante na estratégia saúde da família. Rev. Enf. UERJ., v. 18, n. 4, 2010.

BRASIL. Ministério da Saúde. Guia prático do programa saúde da família. 2001. Disponível em: <http://www.saude.gov.br>. Acesso em: 13 jan. 2012.

Ministério da Saúde. Portaria no 777/GM, de 28 de abril de 2004. Dispõe sobre os procedimentos técnicos para a notificação compulsória de agravos à saúde do trabalhador em rede de serviços sentinela específica, no Sistema Único de Saúde - SUS. Brasília, DF: DOU, 29 abr. 2004. (no 81, Seção 1, pp. 37-38).

Ministério da Saúde. Agência de Vigilância Sanitária - ANVISA. RDC n 50/2002. Dispõe sobre normas destinadas ao exame de aprovação dos estabelecimentos assistenciais de saúde. Brasília, 2002. Disponível em: <http://www.anvisa.gov.br>. Acesso em: 25 jan. 2011.

. Ministério do Trabalho e Emprego. Programa de Prevenção de Riscos Ambientais

NR 15-115.000-6 de 22 de dezembro de 1977. Disponível em: <http://www.mte.gov.br/Empregador/segsau/Legislacao/Normas/>. Acesso em: 20 jan. 2011

Ministério do Trabalho e Emprego. Portaria no 25 de 29.12.94 - Norma regulamentadora - NR9: riscos ambientais. Brasília (DF): Programa de prevenção de riscos ambientais; $1994 . \quad$ Disponível em: <http://portal.mte.gov.br/data/files/8A7C816A47594D040147D1414815672F/NR09\%20(atualizada\%202014).pdf>. Acesso em: 02 fev. 2011.

Consolidação das leis do trabalho. Porto Alegre: Verbo Jurídico; 2005.

Ministério da Previdência Social. Conceito, definições e caracterização do acidente do trabalho, prestações e procedimentos. 2008. Disponível em: $<$ www1.previdencia.gov.br/pg secundarias/paginas perfis/perfil Empregador 10 04- 
A.2005>. Acesso em: 02 fev. 2012.

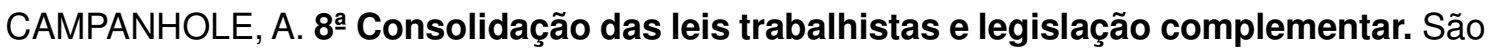
Paulo: Atlas, 2004.

CASTRO, M. R.; FARIAS, S. N. P. A produção científica sobre riscos ocupacionais a que estão expostos os trabalhadores de enfermagem. Rev. Enferm., v. 12, n. 2, 2008.

CHIODI, M. B.; MARZIALE, M. H. P. Riscos ocupacionais para trabalhadores de Unidades básicas de saúde: revisão bibliográfica. Acta Paul. Enferm., v. 19, n. 1, 2006.

COSTA, E. V. Medidas da umidade relativa do ar em uma ambiente fechado. Rev. Bras. Ensino de Física, v. 25, n. 3, pp. 346-8, 2003.

DALRI, R. C. M. B.; ROBAZZI, M. L. C. C.; SILVA, L. A. Riscos ocupacionais e alterações de saúde entre trabalhadores de enfermagem brasileiros de unidades de urgência e emergência. Cienc. y Enfermeria, n. 16, v. 2, 2010.

FARIAS, S. N. P.; ZEITOUNE, R. C. G. Riscos no trabalho de enfermagem em um centro municipal de saúde. Rev. Enf. UERJ, v. 13, n. 2, pp. 167-74, 2005.

LOBIONDO-WOOD, G.; HABER, J. Pesquisa em enfermagem: métodos, avaliação crítica e utilização. 4. ed. Rio de Janeiro: Guanabara Koogan, 2001.

MARINHO, J. Profissão perigo. COREN - SP, v. 52, 2004.

MAURO, M. Y. C. et al. Riscos ocupacionais em saúde. R. Enferm. UERJ; v. 12, pp. 338-45, 2004.

MENDES, K. D. S.; SILVEIRA, R. C. C. P.; GALVÃO, C. M. Revisão integrativa: método de pesquisa para a incorporação de evidências na saúde e na enfermagem. Texto \& Contexto Enfermagem, Santa Catarina, v. 17, n. 4, pp. 758-764, 2008. Disponível em: $<$ http://www.scielo.br/ pdf/tce/v17n4/18.pdf>. Acesso em: 02 set. 2012.

NUNES, M. B. G. Riscos ocupacionais existentes no trabalho dos enfermeiros que atuam na rede básica de atenção à saúde no município de Volta Redonda - RJ. 2009. 169f. Tese (Doutorado) - Universidade de São Paulo, Escola de Enfermagem de Ribeirão Preto, Ribeirão Preto, SP, 2009.

POLIT, D. F.; BECK, C. T.; HUNGLER, B. P. Fundamentos de pesquisa em enfermagem: métodos, avaliação e utilização. 5. ed. Porto Alegre: Artmed, 2004.

POSSO, M. B. S. As fontes de riscos físicos e químicos incidentes sobre os membros da equipe cirúrgica. 1988. Tese (Doutorado) - Escola de Enfermagem da Universidade de São Paulo, São Paulo, 1988.

POSSO, M. B. S.; COSTA, D. S. P. Riscos potenciais que envolvem o trabalho no ambiente hospitalar. Âmbito Hospitalar, São Paulo, v. 2, pp.10- 21, 1998.

POSSO, M. B. S.; SANT'ANNA, A. L. G. G. Riscos físicos e químicos que envolvem o trabalho em centro cirúrgico. In: CARVALHO, R.; BIANCHI, E. R. F. Enfermagem em centro cirúrgico e recuperação. Barueri, SP: Manole, 2007. 
REZENDE, M. P. Agravos à saúde de auxiliares de enfermagem da exposição ocupacional aos riscos físicos. 2003. 127f. Dissertação (Mestrado em Enfermagem) Escola de Enfermagem de Ribeirão Preto da Universidade de São Paulo, São Paulo, 2003.

RIOS, J. C. C.; VARELA, C. D. S. Riscos ambientais em unidade de saúde da família. In: JORNADA CIENTÍFICA E TECNOLÓGICA DO OESTE BAIANO e SEMANA NACIONAL DE CIÊNCIA E TECNOLOGIA. 3, Barreiras, 2010. Anais... Barreiras, BA, 2010.

SÊCCO, I. A. O.; ROBAZZI, M. L. C. C. Acidentes de trabalho e riscos ocupacionais no dia-adia do trabalhador hospitalar: desafio para a saúde do trabalhador. Rev. Espaço para a Saúde, v. 4, n. 1, 2003.

SILVA, M. K. D.; ZEITOUNE, R. C. G. Riscos ocupacionais em um setor de hemodiálise na perspectiva dos trabalhadores da equipe de enfermagem. Rev. Enferm., n. 13, v. 2, 2009.

SILVA, R. C. G.; FELLI, V. E. A. Um estudo comparativo sobre a identificação dos riscos ocupacionais por trabalhadores de enfermagem de duas unidades básicas de saúde do município de São Paulo. Rev. Esc. Enferm. USP, v. 36, n. 1, pp. 18-24, 2002.

SIQUEIRA, F. C. V. et al. Barreiras arquitetônicas a idosos e portadores de deficiência física: um estudo epidemiológico da estrutura física das unidades básicas de saúde em sete estados do Brasil. Ciência e Saúde Coletiva, v. 14, n. 1, 2009.

SOARES, A. D.; COSTA, C. C.; RICARTE, F. M. C. Trabalho em saúde: analisando os riscos ambientais em um Centro de Saúde da Família. In: CONGRESSO BRASILEIRO DE ENFERMAGEM - CBEn. 61, 2009, Fortaleza. Anais... Fortaleza: CBEn, 2009.

SOUSA, J. V.; CAMPOS, L. F. Relato de experiência quanto a orientação de conduta frente a acidentes de trabalho com pérfuro-cortantes e fluidos orgânicos. Cogitare Enferm, Curitiba, v. 13 , n. 4 , pp. 602-606, out./dez. 2008. 